\title{
Giannina Bertarelli, una italiana que traducía en La Habana. Reflexiones sobre vida y obra'
}

\author{
lledys González \\ iledys.gonzalez@uniroma $1 . i \dagger$ \\ https://orcid.org/0000-0001-6275-1393 \\ Università di Roma "La Sapienza", Italia
}

\section{Resumen}

Giannina Bertarelli (Italia, 1921-Cuba, 1994) ha sido citada en muy escasas ocasiones como parte del panorama cultural de Cuba. Sin embargo, fue una figura influyente no solo en la difusión de las literaturas italiana y francesa a través de sus traducciones, inicialmente para la revista Pensamiento Crítico y luego para el Instituto Cubano del Libro, sino también en los comienzos de la enseñanza del italiano en La Habana y en su rol como periodista. El presente trabajo de investigación pretende esbozar primeramente una breve biografía intelectual para rescatar del olvido la memoria de una mujer extraordinaria que llevó una vida novelesca al dejar su país de origen y establecerse de manera definitiva en una Cuba en plena trasformación política y social. La historia de su vida privada define las facetas de su labor como traductora, que serán expuestas aquí. El objetivo esencial de este trabajo es la reconstrucción del corpus traducido por ella y la reflexión sobre los autores extranjeros publicados (Mario de Micheli, Tommaso Landolfi, Giorgio Bassani, Ippolito Nievo y Alba de Céspedes, entre otros) para comprender los perfiles de las obras (temáticas, estilos, géneros) que Bertarelli asumió como traductora. Para ello se ha de dialogar con los estudios precedentes de naturaleza historiográfica y crítica sobre traducción y literatura europea publicada en Cuba.

Palabras clave: historia de la traducción en Cuba, literatura italiana, edición de libros en Cuba, Giannina Bertarelli, estudios transatlánticos.

\section{Giannina Bertarelli. An Italian who translated in Havana. Reflections on her life and work}

\begin{abstract}
Giannina Bertarelli (Italy, 1921- Cuba, 1994) has been cited on very few occasions as part of Cuba cultural panorama. However, she was an influential figure not only in the dissemination of Italian and French literature through her translations for the magazine Pensamiento Critico and then for the Cuban Book Institute, but also as a pioneer in teaching the Italian language in Havana, and as a journalist. This text presents a brief intellectual biography to rescue the memory of an extraordinary woman from oblivion - a woman who led an adventurous life after leaving her country of origin to settle in Cuba at a time of deep political and social changes. The history of her private life defines the facets of her work as a translator, and those facets will be presented here. This work aims essentially at reconstructing the corpus translated by Bertarelli and to reflect upon published foreign authors (Mario de Micheli, Tommaso Landolfi, Giorgio Bassani, Ippolito Nievo and Alba de Céspedes, among others) to understand the profiles of the works (themes, styles, genres). For this reason, it is necessary
\end{abstract}


to enter into dialogue with previous studies of a historiographical and critical nature on translation and European literature published in Cuba.

Keywords: translation history in Cuba, Italian literature, edition of books in Cuba, Giannina Bertarelli, Transatlantic studies.

Giannina Bertarelli, une italienne qui traduisait à La Havane. Réflexions sur sa vie et son travail

\section{Résumé}

Giannina Bertarelli (Italie, 1921-Cuba, 1994) a rarement été reconnue sur la scène culturelle cubaine bien qu'elle ait été une figure influente non seulement dans la diffusion de la littérature italienne et française à travers ses traductions pour la revue Pensamiento Crítico et plus tard pour l'Institut cubain du livre, mais aussi dans les premiers temps de l'enseignement de l'italien à La Havane et dans son rôle de journaliste. Le texte entend d'abord esquisser une brève biographie intellectuelle pour sauver de l'oubli le souvenir d'une femme extraordinaire qui a mené une vie romantique en quittant son pays d'origine et en s'installant définitivement dans une Cuba en pleine transformation politique et sociale. L'histoire de sa vie privée définit les facettes de son travail de traductrice qui seront exposées ici. L'objectif essentiel de ce travail est la reconstruction du corpus traduit par elle et la réflexion sur les auteurs étrangers publiés (Mario de Micheli, Tommaso Landolfi, Giorgio Bassani, Ippolito Nievo et Alba de Céspedes, parmi d'autres) pour comprendre les profils des œuvres (thèmes, styles, genres) que Bertarelli a assumé en tant que traductrice. Pour ce faire, il est nécessaire de dialoguer avec les études antérieures à caractère historiographique et critique sur la traduction et la littérature européenne publiées à Cuba.

Mots clés : histoire de la traduction à Cuba, littérature italienne, édition de livres à Cuba, Giannina Bertarelli, études transatlantiques. 


\section{Introducción}

Advertía hace unos años la eminente estudiosa cubana Luisa Campuzano, de modo oblicuo en ocasión de un homenaje a la intelectual Graziella Pogolotti, sobre la obligada tarea aún pendiente de recordar la encomiable labor de Giannina Bertarelli (1921-1994) como traductora del Instituto Cubano del Libro (ICL) (Campuzano, 2006). En el 2018, fue precisamente Pogolotti quien publicó en la revista Revolución y Cultura una grata memoria sobre la traductora italiana a la que la unían nexos de amistad heredados de su padre, el pintor cubano de origen italiano Marcelo Pogolotti (19021988). Probablemente sea Graziella Pogolotti, quien más haya salvado el nombre de Giannina Bertarelli del anonimato. La ha recordado no solo allí, su más completo tributo, sino desde antes, en otros textos personales referentes a su propia vida y al contexto intelectual habanero del cual participaba y del que formaban parte Bertarelli y su esposo Enrique Collado (19151976) (Pogolotti, 2012; Grant, 2003).

Junto al artículo "Más sobre el ICL y sus traductores. Giannina, persona, contextos", de Graziella Pogolotti (2018), solo puede citarse otro texto que rescata la figura de la traductora, este menos conocido en el ámbito cubano por haber sido publicado en Italia, Cuba Cultura. Viaggio nell'identità di un'isola (Garzia y Fleites, 1997). Ese libro, hecho a cuatro manos por los intelectuales Aldo Garzia y Alex Fleites -italiano y cubano, respectivamente-, constituye una exploración cultural de Cuba, amena y sucinta como guía de viaje que es, pero al tiempo rigurosa en la revelación de datos novedosos, fruto de una evidente investigación profunda. Tal es el caso del capítulo "Italiani a Cuba", bosquejo sobre la presencia permanente o temporal de italianos insignes en la isla, que podría considerarse uno de los pocos estudios generales sobre el tema junto a los ya clásicos libros de Fernando Ortiz Las simpatias de Italia por los mambises cubanos (1905), Los mambises italianos (1909) e Italia y Cuba (1944)-, que ha motivado Italianos por la libertad de Cuba, de Enrique Pertierra Serra (2000), además de la investigación más completa en nueve volúmenes, Emigrazione e presenza italiana in Cuba, de Domenico Capolongo (ed.) (2010), y el recientemente publicado título de Ricardo Rosselló (2019), Presencia italiana en Cuba (1492-1902). Es en dicho apartado del texto de Garzia y Fleites (1997), donde vienen citados Cristóbal Colón, Giovanni Battista Antonelli, Antonio Meucci, Oreste Ferrara e Italo Calvino, que aparece la semblanza biográfica sobre Giannina Bertarelli, derivada, como en el artículo de Pogolotti, de la memoria y de las emociones de su autor Aldo Garzia, amigo cercano de la traductora.

Aparte de estas dos publicaciones la presencia de Bertarelli en el ámbito intelectual cubano parecería nula, si no fuera por esporádicos trabajos sobre traducción donde se cita alguna que otra obra traducida por ella (Bello, 2019; Sánchez Guevara, 2018). Con frecuencia sucede que muchos de los estudios que encontramos sobre la traducción literaria en Cuba prestan atención a casos de escritores traductores y, por tanto, la labor ingente de otros traductores queda aún sin reconocer. No obstante, este juicio no debe ser leído con absolutismo, pues cada año se despliegan exposiciones en el marco de un ya habitual simposio sobre traducción literaria en la Unión de Escritores y Artistas de Cuba (UNEAC), exposiciones que de seguro ayudarán a construir en el tiempo una historia orgánica de la traducción en la isla. En ese volumen que está por escribirse no podrá faltar el episodio que destaque la trayectoria de Giannina Bertarelli, no solo como traductora sagaz, sino también como mediadora cultural entre Italia y Cuba. 
En las líneas que siguen ha de esbozarse, primeramente, una biografía intelectual mínima, ${ }^{2}$ con el fin de arrojar luz sobre el nombre de una traductora casi olvidada - aprovechando el valioso testimonio hablado de su hija Adriana Collado Bertarelli para tal apartado-, ${ }^{3}$ luego ha de desglosarse el corpus de los títulos -basado inicialmente en una búsqueda detallada en los catálogos bibliotecarios y las bibliografías anuales de las publicaciones cubanas - que se dieron a conocer en el país gracias al intelecto y la competencia lingüística de esta figura. La combinación de estos dos cuadros, el biográfico y el bibliográfico, relativos a Giannina Bertarelli, más que un reclamo a los estudios de traducción en Cuba, espera ser umbral de futuras indagaciones en el marco de la recepción de la literatura italiana en la isla y de los diálogos transatlánticos.

2 La biografía intelectual femenina es una línea de investigación muy atendida en los últimos años por la academia italiana, con el objeto de "incorporar" a la historia la producción de las mujeres escritoras, de reinsertar las obras canceladas o mantenidas al margen (Zancan, 2005). Muchas de estas autoras, fundamentalmente las del siglo xx, tuvieron una actuación política, por lo que el énfasis en la red intelectual y la vida pública son ejes prioritarios para comprender a fondo la producción literaria. El grupo de investigación encabezado por la académica Marina Zancan, junto a Monica Storini y Laura di Nicola, entre otras, ha arrojado importantes resultados en la reconstrucción de la memoria de varias autoras italianas a través de trabajos que se encuentran en la colección Scrittici e intellettuali del Novecento, de la editorial Mondadori (Zancan, 2005; Di Nicola, 2012; Rubini, 2019). Esta metodología aplicada aquí a la figura de Giannina Bertarelli pretende un doble rescate para la historia: mujer emigrada, por tanto, desplazada, y además traductora, oficio que como aseveraba Lawrence Venuti ha sido sentenciado a una invisibilidad (Venuti, 1995).

3 Con vistas a este ensayo tuvo lugar una comunicación personal (vía internet, diciembre del 2019) con Adriana Collado Bertarelli, quien refirió abundantes datos sobre la vida de su madre. Ese testimonio oral es la fuente principal para la reconstrucción biográfica que queda expresada en estas páginas.

\section{Giannina Bertarelli, una vida itinerante hasta el hallazgo insular}

Nacida en la ciudad italiana de Milán en 1921, Giannina Bertarelli fue la hija menor de un matrimonio de clase media-baja. Desde pequeña le tocó vivir desventuras que signaron su destino, pero, sobre todo, su personalidad emancipada y resuelta. Siendo una niña perdió a su madre, quien había enfermado de fiebre tifoidea tras cuidar de la hija mayor que, en cambio, sobrevivió a la enfermedad. Años más tarde, la familia se trasladó a Lugano, donde el padre había logrado emplearse como obrero en una fábrica. En Suiza, el padre contrajo segundas nupcias y tuvo otros hijos del nuevo matrimonio. Giannina Bertarelli logró culminar sus estudios de bachillerato y una vez cumplida la mayoría de edad decidió regresar a Milán.

La Segunda Guerra Mundial había llegado a su fin y en Italia se vivía con optimismo un clima de reconstrucción social, caldeado de debates políticos, que se extendía a la cultura, en especial, al campo de la creación literaria. En una de esas tertulias culturales frecuentes en la época, Giannina Bertarelli conoció al escritor Stefano Terra, con quien mantendría una relación sentimental durante largos años. Terra era un intelectual que estuvo comprometido con la Resistencia en Turín, su urbe natal, vinculado al grupo Giustizia e Libertà, junto a Leone Ginzburg y Cesare Pavese, y luego exiliado a E1 Cairo, donde continuó su campaña antifascista con otros italianos, como la escritora Fausta Cialente. En la Italia de la posguerra Terra colaboró como periodista con diferentes boletines y fue enviado al extranjero como corresponsal de Ansa (Agenzia Nazionale Stampa Associata), La Stampa y la RAI. En sus estadías en el exterior lo acompañó Giannina Bertarelli: de ese modo pasaron unos tres años en París, luego en Belgrado (de la antigua Yugoslavia) y finalmente en Atenas. De aquella etapa es preciso 
enfatizar el primer contacto de Bertarelli con contextos lingüísticos diversos, su aprendizaje del francés, y quizás su primer trabajo como periodista.

E1 12 de septiembre de 1953, se publicó en $L a$ Stampa, el artículo "Avventure di giornalisti in un paese che non li ama", de Vittorio Gorresio (1953), en el que se narra la detención de Terra en la Yugoslavia de Josip Broz Tito, acusado de varios delitos mediante los que se pretendía obstaculizar su trabajo como periodista. Las colaboraciones desde este país pretendían hacer conocer en Italia las ideas de Tito y los cambios generados hacia el socialismo en los Balcanes. Como se explica en el texto, Terra y su compañera habían logrado introducir una grabadora con la que registraban los discursos de Tito por la radio local — que por la clausura del país no se difundían en el exterior - para luego hacerlos traducir y elaborar la noticia en Italia. Al ser apresado Terra, la embajada italiana contactó a Giannina Bertarelli para que se refugiara allí; sin embargo, la respuesta de ella fue la de una mujer intrépida y tenaz: culminar la tarea de periodista iniciada por el marido. Y así, como precisa Gorresio, "fece tradurre tutti i nastri e poi, ma solo allora, andò alla Legazione a consegnarli perchè fossero al sicuro" (1953, p. 5). ${ }^{4}$ Tras la liberación de Terra, la pareja regresó a Italia, donde fueron recibidos con admiración, y luego viajaron a Grecia. Aquella estancia, siempre de colaboración periodística, sería la última que pasarían juntos: la relación terminó y Giannina Bertarelli regresó a Milán, donde intentó inútilmente encontrar para sí un espacio profesional en un sector intelectual dominado por los hombres. Así surgió en ella la idea de irse lejos, a un lugar donde no solo pudiera librarse del mal de amor, sino también emanciparse definitivamente.

4 "Hizo traducir todas las cintas y luego, solo entonces, fue a la Embajada a entregarlas para que estuvieran al seguro" (traducción de la autora).
A La Habana, Giannina Bertarelli llegó en barco en 1956 con la idea de solicitar un visado que le permitiera ingresar a México, meta de su travesía. Sin embargo, el descubrimiento de la ciudad habanera de los cincuenta, tan cosmopolita, moderna y a la vez caótica en su herencia colonial, la fascinó por la maravilla americana, una fascinación de la que no pudo escapar. Bertarelli decidió ponerse en contacto con la comunidad italiana residente en $\mathrm{La}$ Habana y conoció al pintor cubano Marcelo Pogolotti - hijo del italiano emigrado a Cuba en 1898 Dino F. Pogolotti-, quien, junto a su hija Graziella, se convertiría en su principal familia de acogida. Así las tertulias en la casa de los Pogolotti se volvieron habituales: se hablaba de la cultura francesa, de literatura y arte, pero a la vez se hablaba de Revolución. Eran los años de la lucha clandestina en La Habana y el clima de inestabilidad política y de represión parecía condicionar su partida, pero Bertarelli postergaba siempre su viaje. Se había establecido y logrado sus primeros trabajos de traducción al español: un manual de mecánica de la Fiat para una fábrica habanera y un manual de fertilizantes. Vale decir que el español lo aprendió in situ y que un gran mérito de su obra posterior como traductora fue el desafío de trasladar un texto hacia una lengua que no era la propia, invirtiendo la lógica fundamental del oficio, que en algunos casos en ella se hacía doblemente asombroso: al traducir del francés al español. Por otro lado, Giannina Bertarelli había continuado colaborando para la prensa italiana (Ansa y la $R A I$ ) sobre la realidad cubana que en 1959 se había vuelto la principal noticia en todo el mundo. El periodismo fue una actividad que mantuvo a lo largo de su vida y que representaba un puente directo entre esos dos mundos a los cuales pertenecía, una forma de confrontar esos espacios distantes, Italia y Cuba.

En aquellos primeros años en la isla, Bertarelli se enamoró de Enrique Collado, un prestigioso 
psiquiatra, amigo de los Pogolotti, que había sido esposo de Eva Fréjaville, la musa del pintor Carlos Enríquez. La pareja se casó en 1964 y estableció su residencia en el Vedado, entre las calles de Línea y Paseo, en un apartamento que sería visitado por escritores como Italo Calvino, Virgilio Piñera y Alejo Carpentier. La amistad de Bertarelli con Piñera fue muy cercana y se fortaleció cuando ambos coincidieron como trabajadores del Instituto Cubano del Libro (ICL), donde el poeta cubano, silenciado por el sistema, había quedado recluido haciendo trabajos de edición y traducción del francés. Para el ICL, Bertarelli trabajó durante dos décadas, prácticamente desde su fundación en 1967 hasta principios de los ochenta. El conjunto de obras traducidas por ella (del francés y del italiano) es muy variado; lo integran desde textos de matriz filosófica, política, literaria hasta de crítica de arte y lingüística. Pero antes de consagrarse como auténtica y prestigiosa traductora para el Instituto, se había ejercitado con la revista Pensamiento Crítico, en la que encontramos las primeras pruebas de su oficio. En aquella época, fundó además la enseñanza del italiano en la Academia de idiomas "Abraham Lincoln" en La Habana (Pogolotti, 2018).

En las últimas dos décadas de su vida, Giannina Bertarelli abandonó la traducción literaria y se dedicó exclusivamente al periodismo, haciendo reportajes para Italia a través de $A n s a$. Como apuntan Aldo Garzia y Alex Fleites en su libro, a raíz de una entrevista, la traductora italiana vivió a partir de los ochenta un cierto descontento por el sistema cubano:

Per molti anni è stata la corrispondente attenta e puntuale dell'agenzia di stampa Ansa, oltre che il punto di riferimento indispensabile per tutti i giornalisti italiani che partivano verso Cuba disorientati e sperduti [...]. Negli anni Ottanta quella giornalista italiana inizia a guardare con maggiore distacco alle vicende politiche dell'isola. Restava intatta la passione per il lavoro di cronista. L'interesse per la letteratura l'aveva sempre accompagnata durante tutta la sua vita. ${ }^{5}$ (Garzia y Fleites, 1997, p. 211).

La suya fue una vida itinerante y novelesca, movida por el amor y el compromiso político, hasta dar con un hallazgo insular que motivara su permanencia definitiva. En Cuba tuvo una hija cubana y logró emanciparse profesionalmente en una época de grandes transformaciones sociales, pero que tanto aportó a la liberación de la mujer. Giannina Bertarelli falleció en La Habana en 1994 sin nunca escribir sus memorias.

\section{Traducciones para la revista Pensamiento Crítico}

Es difícil aseverar, sin correr el riesgo de cometer un error futuro, cuál fue el primer trabajo de traducción publicado por Giannina Bertarelli. Pero ante la necesidad de determinar un inicio en su trayectoria podría citarse entre esos primeros títulos un texto que, sin embargo, no traduce de su lengua materna, sino del francés, que había aprendido bien durante los años vividos en París. Se trata de "El intelectual frente a la Revolución”, de Jean-Paul Sartre (1968), para la revista mensual Pensamiento Crítico, el 9 de octubre de 1968.

Entre los temas de resonancia en la revista se encontraban algunos dedicados a la Revolución cubana y a sus fundamentos ideológicos,

5 "Durante muchos años fue la corresponsal atenta y puntual de la agencia de prensa $A n s a$, además de punto de referencia para todos los periodistas italianos que viajaban a Cuba desorientados y perdidos [...]. En los años ochenta aquella periodista italiana comenzó a observar con mayor desapego los acontecimientos políticos de la isla. Permaneció intacta su pasión por el trabajo como cronista. El interés por la literatura la acompañó durante toda su vida" (traducción de la autora). 
reflexiones marxistas, aspectos teóricos de la lingüística y el arte, el nuevo cine cubano, el movimiento surgido en mayo de 1968 en Francia, el movimiento de los derechos civiles y el movimiento negro en Estados Unidos, los movimientos estudiantiles en Alemania e Italia, entre otros argumentos vinculados a la sociedad y al pensamiento, siempre con un corte teórico y crítico (Kohan, 1993). En este plural abanico temático no podían quedar por fuera las ideas de uno de los filósofos más notables de la época.

Jean-Paul Sartre, quien ya había estado en Cuba durante un viaje a Centroamérica en 1949, se interesó de inmediato por el fenómeno de la Revolución en su posición de crítica al colonialismo y aceptó la propuesta oficial del nuevo gobierno, a través de Carlos Franqui, de hacer una visita al país en 1960. En esa famosa y conocida ocasión viajó acompañado de Simone de Beauvoir, y se reunió con Fidel Castro, Che Guevara y otros líderes e intelectuales comprometidos con el proceso. Tras una experiencia cubana que viviera con cierto enamoramiento, Sartre escribió entonces una serie de dieciséis artículos para el periódico France Soir con el título de Ouragan sur le sucre y que aparecieron luego en un volumen independiente en español, Huracán sobre el azúcar (Sartre, 1960a), y en inglés, publicado como Sartre on Cuba (en Paolucci, 2007). ${ }^{6}$ Durante su estancia en la isla, inspirado por diálogos diversos y, en especial, por un conversatorio con estudiantes de la Universidad de La Habana, Sartre se dedicó a escribir "Ideología y revolución" (1960b), ensayo publicado en español por la revista Lunes de Revolución, bajo las siglas del traductor J. J. A.

El texto de Sartre traducido por Bertarelli fue tomado de una entrevista titulada L'intellectuel face à la révolution publicada en el periódico Le

6 Véase Hewitt, 2007.
Point en enero de 1968. Aquí se define el intelectual de izquierda que, a juicio del filósofo, es la categoría que solo puede asimilar el sentido completo de la palabra 'intelectual', entendida como un componente de un "grupo socioprofesional que podría llamarse los teóricos del saber práctico" (Sartre, 1968, p. 191). El texto se va adentrando cada vez más en la comprensión del rol del intelectual frente al radicalismo, por lo que tras abordar los casos de China y la uRss llega a Cuba. "Para un intelectual es imposible no ser pro-cubano", aseveraba Sartre (1968, p. 200) aún con el mismo entusiasmo que reflejaba en su texto anterior de 1960, publicado en Lunes de Revolución. E1 diálogo entre ambos títulos es más que evidente. La traducción que se muestra es límpida y fluida, no entorpece la lectura y mantiene la nota reflexiva sobre la discusión.

Otros dos textos de Jean-Paul Sartre aparecen en Pensamiento Crítico (1967, marzo-abril), "E1 pensamiento político de Patricio Lumumba" (1967) y "África del Sur: centro del fascismo" (Sartre, septiembre de 1969); sin embargo, no encontramos el nombre del traductor. Esta omisión se da en muchos otros casos de la revista, donde el texto teórico extranjero declara la fuente en lengua original, pero deja la duda sobre cómo, o mejor dicho, por quién fue trasladado al español. Y hablamos de varias publicaciones tomadas de fuentes diversas, entre ellas los boletines Les Temps Modernes, fundada por Sartre y Simone de Beauvoir, así como New Left Review, de Reino Unido, y Monthly Review, de Estados Unidos. ${ }^{7}$

\footnotetext{
7 En su estudio "Una mirada métrica a la revista Pensamiento Crítico", Vilma Ponce Suárez (2007, p. 138) contabiliza las fuentes extranjeras que sirvieron para dar actualidad a la revista: "En total se tomaron 87 trabajos de 38 revistas de países como: Chile, Perú, Uruguay, Colombia, México, Brasil, Bélgica, Italia, Francia, Inglaterra, África del Sur, Viet Nam y Estados Unidos. Las más frecuentes fueron: Les Temps Modernes — 8 tra-
} 
Otro ensayo es traducido por Giannina Bertarelli en el mismo número de la revista (1968, 9 de octubre): "Las contradicciones del capitalismo tardío, los estudiantes antiautoritarios y su relación con el Tercer Mundo", de Rudi Dutschke. Se trata de un largo texto (cerca de cien páginas) que vendría siendo un inmediato homenaje a su autor, líder estudiantil alemán, que el 11 de abril del mismo año había sido víctima de un grave atentado por sus ideas marxistas (Dutshke, 1968). En este caso, no se declara la fuente original que sirviera de soporte a la traducción que, en cambio, sí aparece firmada por Bertarelli. Evidentemente, dadas las competencias lingüísticas de la traductora, el texto de base no debió haber sido en alemán, sino en una de las lenguas conocidas por ella como puente al español. Sin embargo, ante la ausencia de un dato escrito solo quedan las conjeturas.

Otro título del francés traduce Giannina Bertarelli para Pensamiento Crítico: de Louis Althusser, "Lenin y la filosofía" (1969, 11 de noviembre, pp. 120-153). Aquí sí se conoce la fuente: una ponencia presentada ante la Sociedad Francesa de Filosofía, el 24 de febrero de 1968, y que se publicó en Cuba con el consentimiento del presidente Jean Wahl, como viene indicado a pie de página. En este trabajo, que apareció publicado posteriormente también en español como volumen independiente, su autor expone los aportes del pensamiento de Lenin a la teoría marxista. Fernando Martínez Heredia refería en "Althusser y el marxismo" (1970), también para Pensamiento Crítico, algunas claves sobre las discusiones que en el contexto cubano generaban las ideas del filósofo francés. ${ }^{8}$

bajos, New Left Review —6 trabajos, The Socialist Register -5 trabajos, Monthly Review — 5 trabajos y Partisans - 4 trabajos".

8 Véase Ponce Suárez (2019) para más noticias sobre los autores franceses publicados en Pensamiento Crítico.
Es importante reconocer el valor de estos trabajos traducidos por Giannina Bertarelli, representativos del intenso debate de ideas en el panorama intelectual de la época. Por otro lado, en el plano personal, esta experiencia inicial demostraría su madurez en el acto de traducir y su capacidad para asumirlo ya como un oficio establecido. Pensamiento Crítico sería entonces el camino para llegar a desempeñarse como traductora del Instituto Cubano del Libro.

\section{La obra de una traductora}

Un primer paso en el desarrollo de la industria del libro en Cuba después de 1959 fue la concepción de la Edición Revolucionaria en 1963, que pretendía, para una difusión colectiva del saber, la fotocopia del libro impreso, lo cual eliminaba los derechos de autor y permitía la reproducción de las obras más importantes en el ámbito internacional, ya fueran de carácter científico-técnico o literario. Así surgieron los textos universitarios y la colección de literatura universal del sello Huracán. Como precisaba Pamela María Smorkaloff en su exquisita investigación Literatura y edición de libros. La cultura literaria y el proceso social en Cuba (1987), se trataba de una industria editorial no comercial. ${ }^{9}$

9 Fernando Martínez Heredia, uno de los directores de la Edición Revolucionaria, que luego sería fundador de la editorial de Ciencias Sociales, declaraba sobre tal experiencia:

En aquellos años 'fusilamos' los derechos de autor de un gran número de libros extranjeros que publicamos. Es decir, no les pagábamos nada. Ahora se estima que los derechos de autor son una de las cuestiones fundamentales del capitalismo actual, y numerosos especialistas estudian ese tema. Nosotros los fusilamos, sencillamente, y no pasó nada. Entre otras cosas, porque nadie tenía ninguna soberanía sobre nosotros. Algún provecho le sacamos a ser un pequeño país libre. Buscamos lo más reciente de las ciencias y lo publicamos en Cuba. Los alumnos y los 
A raíz de esa experiencia se fundó en 1967 el Instituto Cubano del Libro (ICL). Este abarcaba todas las nuevas editoriales (Arte y Literatura, Ciencia y Técnica, Ciencias Sociales, Pueblo y Educación, entre ellas) y se ocupaba de la planificación, producción y distribución de sus publicaciones.

E1 ICL contaba además con un Departamento de Traducciones, que pretendía cubrir las solicitudes editoriales. Entre las lenguas de las que se traducía estaban el inglés, el italiano, el francés, el ruso, el portugués y el polaco. Además, se acogían libros de muy variadas materias, tanto literarias como científicas y sociales. La importancia de este departamento la explica Olga Sánchez Guevara, quien fuera traductora del centro:

Un enclave intercultural en el corazón de La Habana: eso fue el Departamento de Traducciones, cuya historia merecería una investigación a fondo, en la que se destaque la contribución de los traductores del Departamento a la divulgación de las literaturas del Tercer Mundo y Europa del Este, de las cuales se publicaron en Cuba muchas obras en primeras (y a veces únicas) traducciones al español. Antes de recibir sus Premios Nobel, los africanos Wole Soyinka y Nadine Gordimer eran conocidos al lector cubano, al que también le eran familiares nombres como Chinua Achebe, Usmán Sembén, Mohammed Dib, Aziz Nesin, Karel Capek e Ismail Kadaré, entre otros. (2018, p. 21)

El Departamento de Traducciones actualmente ha dejado de existir, pero la historia de su trascendencia cultural para el contexto hispanoparlante está en continuo rescate (Sánchez Guevara, 2018).

profesores cubanos dieron un salto tremendo en sus posibilidades y conocimientos. (2008, p. 242)
La labor de Giannina Bertarelli como traductora para el ICL se desarrolló durante los años setenta y ochenta; sus títulos se encuentran en el sello de Ciencias Sociales, así como en Arte y Literatura. También puede apreciarse su trabajo en la revisión y cotejo de traducciones, pues como asevera Olga Sánchez Guevara, "no sólo se traducía, sino también se revisaban traducciones, ajenas y propias; durante largo tiempo esta tarea se realizaba a dúo entre dos traductores de la misma lengua, un estilo que abreviaba el tiempo de revisión y beneficiaba al traductor menos experimentado" (2018, p. 21).

Para abordar de modo inmediato un catálogo razonado de los libros traducidos por Giannina Bertarelli debe hablarse en términos de corpus en construcción, no permanente, pues la exploración sobre los títulos publicados aún no ha concluido y en el futuro podría arrojar nuevos datos. Aunque en estas páginas se quisiera hacer énfasis en la divulgación de la literatura italiana en Cuba, dado que el italiano era la lengua materna de la traductora, no debe solaparse, sin embargo, el valor de las obras traducidas del francés, por lo que también tendrán un espacio significativo aquí. E1 examen puede esbozarse en dos grupos textuales: los teóricos primeramente y los literarios luego.

\subsection{Libros teóricos traducidos}

El primer libro que Giannina Bertarelli tradujo completamente (al español del italiano) fue Las vanguardias artísticas del siglo $X X$, de Mario de Micheli, publicado en 1967 por Ediciones Unión y tomado de Le avanguardie artistiche del Novecento (Milán: Schwarz Editore, 1959). ${ }^{10} \mathrm{El}$ volumen de casi quinientas páginas en donde

10 Ella firma Giannina de Collado, asumiendo el apellido del esposo. 
el crítico teoriza sobre los "ismos" y compila manifiestos, documentos y programas de movimientos como el Surrealismo, el Dadaísmo y el Cubismo sigue siendo hoy un título de consulta primordial para los estudiosos del arte. En el "Prefacio a la edición cubana", escrito en Milán el 25 de noviembre de 1966, Mario de Micheli expresa las motivaciones de la escritura del texto y su consentimiento para que se traduzca y circule su libro en la isla, a pocos años de la primera publicación italiana:

Debería decir enseguida por qué estoy contento de que este libro mío se publique ahora en Cuba. No me es difícil decirlo. Cuba es un país libre y revolucionario, donde también la cultura se está moviendo en un espacio histórico verdaderamente nuevo; precisamente aquel que toda verdadera vanguardia buscó y busca para ahondar en él sus raíces y para vivir. Quisiera decir que, de cierto modo, el lector cubano puede ser el lector ideal de estas páginas.

[...] las razones más precisas que me indujeron a escribir este tomo nacieron sobre todo de la voluntad de aclarar — contra la liquidación total y apresurada de las vanguardias por parte de una crítica dogmática y, al propio tiempo, contra una celebración de las vanguardias, igualmente genérica y superficial, en clave de gusto- los verdaderos motivos fundamentales que dieron origen a su rebelión, subrayando su validez histórica y su herencia, más allá de los mil disfraces de estetismo. (1972, p. IX)

Muy probablemente el motivo de esta publicación cubana surgiera durante o poco después de la visita del estudioso italiano al país en 1966 para participar como jurado en el Premio de Literatura UNEAC. Las vanguardias artísticas del siglo XX encontrará otra edición cubana en 1972 por el Instituto Cubano del Libro. Y fuera de Cuba puede hallarse traducido al español por Ángel Sánchez Gijón para Alianza Editorial (Madrid, 1979) que ha contado con disímiles ediciones.
En 1970 el nombre de Giannina Bertarelli aparecía en el volumen del importante filósofo húngaro Georg Lukács, Historia y conciencia de clase (Geschichte und Klassenbewußtsein), publicado por Ciencias Sociales con una tirada de cinco mil ejemplares. La traducción llevada a cabo por Francisco Duque proviene del francés Les Éditions de Minuit (París, 1957) que a su vez había sido traducido del alemán por Kostas Axelos y Jacqueline Bois. El cotejo realizado por Bertarelli fue con la traducción italiana de Giovanni Piana para Sugar Editore (Milán, 1967), donde se presentaba un prefacio escrito por Lukács específicamente para dicha edición en el que hacía una revisión crítica de sus trabajos, y que se incluyó entonces en el volumen cubano (pp. 7-33). Este fragmento, aunque no quede allí explícitamente declarado, fue traducido del italiano por ella. Historia y conciencia de clase también incorpora una nota de la editora Teresa Blanco en la que se expone una presentación del autor y de sus ideas.

En 1975, Bertarelli concluyó dos libros para Ciencias Sociales. Primeramente, las Cartas a Kugelmann (Marx, 1975), de Karl Marx, que tradujo del francés de la edición de Éditions Sociales (París: 1971) realizada por el traductor Gilbert Badia, y que fue cotejada por José Rovira Fernández con la edición alemana Marx Engels Werke (Berlín: Dietz Verlag, 1973). Se trata de un volumen de trescientas páginas que presenta la serie epistolar (18621874) de Marx dirigida al médico Kugelmann, que le ayudó a difundir El capital en Alemania. Por otro lado, encontramos Armar a las masas revolucionarias, construir el ejército popular (263 pp.), del vietnamita Vo-Nguyen-Giap (1975), periodista y ministro del Interior durante la presidencia de Ho Chi Minh. Aquí quedaron expuestas tesis marxistas y leninistas sobre la organización militar del proletariado. Sin embargo, se desconoce la fuente que sirvió de 
base a la traducción. Un último título teórico traducido por Giannina Bertarelli para Ciencias Sociales en la década del setenta, junto a Pedro Arce y Mario Díaz Godoy: Los socialistas anteriores a Marx (1976), del historiador italiano Gian Mario Bravo (1976), quien registra aquí una exploración del socialismo de 1789 a 1848 en Francia, Inglaterra, Alemania, Estados Unidos, Italia, entre otros países. Este equipo de traductores trabajó con la edición francesa de 1969 (París: François Maspero), trasladada del original de 1966 (Roma: Editori Riuniti) por Alice Théton.

De la década de los ochenta el primer título teórico traducido por Bertarelli que encontramos es La estética del film (Bálazs, 1980) (212 pp.), del húngaro Béla Bálazs, con prólogo del italiano crítico de cine Umberto Barbaro. Este trabajo para la Editorial Arte y Literatura lo realizó Bertarelli a partir de la edición italiana (Roma: Edizioni di cultura sociale) de 1954.

En 1982 Giannina Bertarelli llevó al español, muy posiblemente a partir de la edición italiana trasladada del inglés por Elena Bona en 1971 (Milán: Longanesi), ${ }^{11}$ El indio Gerónimo: memorias, recopiladas por S. M. Barrett (190 pp.). ${ }^{12} \mathrm{La}$ publicación por Ciencias Sociales comprendía además de la explicación preliminar de Barrett sobre cómo nació el libro (incluida en la primera tirada), un preámbulo tomado de la edición italiana de Frederick W. Turner, profesor de la Universidad de Massachusetts, y un prólogo ad hoc de Carlos Díaz. Se trata de un título importante para la serie etnológica de la editorial, pues constituyó la

11 Con el título Geronimo, la sua storia.

12 Hablo en términos de probabilidad, pues no he podido acceder al ejemplar publicado. La ficha técnica que ha servido a precisar esta publicación proviene de la Bibliografia Cubana (1982). autobiografía hablada de Gerónimo (18291909), último líder de los apaches chiricahuas, que contó a Barrett, en español y que luego él vertió al inglés. ${ }^{13}$ En el 2008 la editorial madrileña Mono Azul publicó otra traducción española de este libro, con el título de Soy apache, del traductor Javier Lucini. El desconocimiento de la publicación cubana y en especial del trabajo de Bertarelli conllevaron a la prensa a señalar erróneamente esta como la primera traducción al español. ${ }^{14}$

En la Bibliografia cubana de 1985, publicada por la Biblioteca Nacional de Cuba "José Martí", se registró la traducción de Giannina Bertarelli del volumen de trescientas páginas Ideas y métodos de la lingüística estructural contemporánea, del lingüista ruso Iuri Apresian (1985) (Ciencias Sociales). Sin embargo, al no tener acceso al ejemplar impreso es imposible identificar la fuente (específicamente, el idioma) que le sirviera a Bertarelli para traducir, pues no conocía la lengua rusa, así que se descarta una traducción del libro original.

En 1989 apareció otro trabajo de la traductora italiana, esta vez del francés, para la editorial Arte y Literatura: Edgard Varèse (301 pp.), de Fernand Ouellette (1989), con prólogo de Leonardo Acosta. Se trataba de una biografía del músico francés Varèse (1883-1965), quien compuso en los Estados Unidos gran parte de su obra, como Octandre e Intégrales. La biografía que traducía Bertarelli, del escritor canadiense Fernand Ouellette (Montreal: Éditions Seghers, 1966) habría de tener una impronta en la ensayística y en la vida del narrador cubano

13 Con la aprobación del presidente Theodore Roosevelt, las memorias se publicaron en inglés con el título Geronimo's Story of His Life (Nueva York: Duffield \& Company, 1906).

14 Véase "Editan por primera vez en español las memorias del indio Gerónimo” (ABC, 2009). 
Alejo Carpentier, quien dejaría una formidable y personal reseña sobre el compositor francés, a quien había conocido durante sus años parisinos. En la semblanza que publicara en Le Nouveau Commerce (1980), reunida luego junto a otros escritos en su libro La novela latinoamericana en vísperas de un nuevo siglo y otros ensayos (México: Siglo Veintiuno Editores, 1981), Carpentier precisaba: "En 1926 tuve el privilegio de escuchar, en México, su Octandre, dirigido por Carlos Chávez durante un concierto de música contemporánea. [...] Desconocido en Europa, Varèse era en los Estados Unidos pieza de escándalo" (1984, p. 240). La memoria narrada por Carpentier con los vuelos de una escritura que asume unas veces cariz novelístico y, otras, agudo sentido de crítica musical podría ponerse en diálogo con el volumen traducido por Bertarelli, amiga además del escritor cubano. ¿Quién sabe si fuera acaso el mismo Carpentier quien algún día le sugiriera la traducción de este título?

\subsection{Traducción de la literatura italiana}

El primer conjunto de obras traducidas por Bertarelli para el ICL, al tratarse de textos teóricos o de crítica y filosofía, entraba fundamentalmente en el sello de la editorial Ciencias Sociales. En la segunda etapa de trabajo para el Instituto se aprecia ya su labor como traductora propiamente literaria del italiano. Los títulos se encuentran formando parte del catálogo de Arte y Literatura durante la década de los ochenta. Sin embargo, la primera novela que traslada al español es de 1977: El día de la lechuza (128 pp.), de Leonardo Sciascia (1977). En su idioma original Il giorno della civetta (Turín: Einaudi, 1961), es una novela detectivesca que transcurre en Sicilia y que pudiera ponerse a discusión con las obras sobre el inspector Montalbano del escritor contemporáneo, recientemente fallecido, Andrea Camilleri.
En 1980 aparecieron de modo simultáneo publicados tres títulos con la traducción de Bertarelli, fruto del trabajo de años precedentes. Las dos solteronas (Le due zitelle), de Tommaso Landolfi (1980), relato o novela breve publicada en 1946 en Bompiani, que trata de dos solteronas y un mono llamado Tombo. Natalia Ginzburg definía muy bien la esencia del libro en su ensayo "Lectura de Landolfi":

El mono Tombo, muy querido por las dos solteronas, huye por la noche de su jaula y comete sacrilegios en el vecino convento. Las dos solteronas, por obediencia a las autoridades eclesiásticas, deben resignarse a matarlo. [...] En la narrativa de Landolfi, los animales representan el misterio de la existencia. Las dos solteronas ignoran por qué Tombo, el amado animal, ha querido realizar el crimen, comerse las hostias consagradas y burlarse y ensuciar los objetos litúrgicos: él está inmerso en el silencio animalesco. (2016, p. 423)

El único libro de literatura infantil traducido por Bertarelli de las obras identificadas es Aventuras de Cebollino (Le avventure di Cipollino, en Editori Riunuti, 1959), del gran narrador Gianni Rodari (1980), que se publicó en 1980 por el sello Gente Nueva, dedicado exclusivamente al género para niños y jóvenes. Este título, con ilustraciones de Rosa Salgado, se reeditó en Cuba en 1998. De Rodari puede decirse que es uno de los autores italianos más leídos y traducidos del siglo xx. En España, por ejemplo, se le suele publicar con recurrencia.

También en 1980 apareció en el catálogo de la Editorial Arte y Literatura la serie de relatos ambientados en la Resistencia en Piamonte en la que participó su autor, Los veintitrés días de la ciudad de Alba (I ventitré giorni della città di Alba), de Beppe Fenoglio (1980), publicado por Einaudi en 1952. El volumen conformado por una docena de cuentos, todos sobre la disputa por recuperar la ciudad de Alba inmersa en la lucha antifascista, constituyó uno de 
los primeros títulos en la época en abordar la crónica partisana. En orden de lectura encontramos primeramente el relato que le dio título al libro, "La salida", "El ardid", "Los inicios del guerrillero Raoul", "Viejo Blister", "Otro muro", "Ettore se va a trabajar", "Aquella antigua muchacha", "El agua verde", "Nueve lunas", "El olor de la muerte", y "Lluvia y la novia". Además, el libro llevaba un prefacio, "Al lector", que muy probablemente fuera escrito por Bertarelli, pues en una publicación de 1981 firmó un preámbulo con el mismo título. En la introducción, donde quedaba resumido un cuadro biográfico del autor sobre su particular implicación en la lucha antifascista, se resumían los temas vitales que abordaban los cuentos:

El relato de la ocupación y posterior pérdida por un destacamento guerrillero, de la ciudad de Alba (tema que retomará en otro libro publicado póstumamente, Il partigiano Johnny) convierten a Fenoglio en el poeta de la guerrilla italiana. Y ello es así porque el escritor ha despojado a su prosa de todo alarde estilístico para ofrecernos, en cambio, con gran sentido del realismo crítico, un impresionante cuadro del momento histórico que le tocó vivir. La épica de Fenoglio, en la que alternan la heroicidad y la claudicación, la violencia y la ternura, la firme decisión y la inconsecuencia, es la épica de un pueblo que, sojuzgado brutalmente por el fascismo durante más de veinte años, desborda sus pasiones para lanzarse, en el momento decisivo, a la lucha por su libertad. (s/a, 1980, pp. 5-6)

El texto introductorio se expande hacia un recuento de los principales sucesos de la Italia nazi y del auge del movimiento antifascista que incluía tres frentes diversos: los comunistas, los socialistas y Giustizia e Libertà. Queda sintetizado de modo coherente y argumentativo el convulso contexto italiano al que perteneció Fenoglio y del cual su obra era genuino reflejo, para que el lector cubano comprendiera mejor los conflictos expuestos en la narración.
Es posible que la publicación del libro surgiera por la propuesta de la propia Bertarelli, tan cercana al argumento tratado en esas páginas, pues había vivido ella misma junto a un escritor que luchó por la liberación de Italia. Siendo un título que se situaba dentro del género de una literatura testimonial y comprometida obtuvo una recepción ideal en Cuba, donde había fecundado en los años sesenta una narrativa de carácter realista, volcada hacia las transformaciones sociales y los episodios de la guerra en la clandestinidad y la guerrilla.

La madurez de Giannina Bertarelli en su oficio como traductora literaria se reveló en 1981 con la publicación de dos títulos de suma importancia. Por primera vez asumía el rol de antologadora de la narrativa moderna italiana, junto al menester de traducir, y ello la connotó definitivamente como una voz de autoridad. Su mano y su intelecto no quedaron solo para trasladar a otro idioma un libro ya concebido por su autor, sino para proponer de sus lecturas, de sus investigaciones literarias, un nuevo libro, resultado de su personal selección y juicio crítico. Así encontramos la antología de cuento Una noche del 43 y otros relatos (Editorial Arte y Literatura) que componían entre otros autores Giorgio Bassani, Luigi Compagnone, Dino Buzzati, Natalia Ginzburg, Anna Maria Ortese, Milena Milani, Leonardo Sciascia y Giusseppe Berto (1981) en una totalidad de cuatrocientas páginas. ${ }^{15}$ Giannina Bertarelli

15 "Una noche del 43”, de Giorgio Bassani; "Los jugadores", de Luigi Compagnone; "La expedición”, de Domenico Rea; "Una muchacha asomada a un balcón”, de Pasquale Festa Campanile; "La inquilina" y "Háblame, dime algo", ambos de Manlio Cancogni; "Los siete mensajeros", de Dino Buzzati; "La madre", de Natalia Ginzburg; "Isla del Ángel”, de Giuseppe Dessi; "Excursión dominical", de Carlo Montella; "Casanova", de Luigi Santucci; "Un par de gafas", de Anna Maria Ortese; "Casa ajena”, de Silvio D’Arzo; "Una velada inolvidable", de Vitaliano Brancati; "El retrato", de Milena 
escribió una breve introducción al volumen ("Al lector"), donde resumía una historia de la narrativa italiana, en particular, para entender la cuentística del siglo xx, de la que se eligió un muestrario en la publicación. Quedaron expresadas allí también las temáticas recurrentes de los relatos y la presentación de los autores más distinguidos, aunque en cada cuento aparecía una nota con el nombre del traductor, la fuente del texto y las publicaciones esenciales del escritor, así como aspectos biográficos mínimos. Como decía la nota de contracubierta, escrita por Bertarelli, pues algunas ideas coinciden con lo dicho en su prefacio:

Cualquier selección de cuentos italianos del período posterior a la Segunda Guerra Mundial es harto difícil, pues en los últimos treintaicinco años este género ha alcanzado un considerable desarrollo y una extraordinaria calidad. Y más ardua resulta la tarea si se pretende cumplir un propósito didáctico con nuestros lectores, poner en sus manos una muestra de autores de gran valía, con ejemplos elogiados por la crítica, pero prácticamente desconocidos en nuestro país [...].

Por lo tanto, presentamos en este volumen una amplia gama temática, donde la guerra, el amor, la ironía, el humor, el ridículo, la épica, lo fantástico, lo simbólico y lo real se conjugan para ofrecer a ustedes las más representativas vertientes de la narrativa breve contemporánea en Italia (Bertarelli en Bassani et al., 1981).

Es notable el balance que hiciera en la selección de los autores, unos más conocidos que otros, y donde no escapan nombres femeninos que sin duda pertenecen a una literatura moderna ya canonizada, como Ginzburg y Ortese. En su estudio "Golondrinas que hacen discretos veranos: antologías contemporáneas de cuentos italianos y cubanos",

Milani; "La muerte de Scarandogi”, de Niccolò Tucci; "Giufá", de Leonardo Sciascia; "Tía Bessy, in memoriam", de Giuseppe Berto.
Mayerín Bello se refiere a ese volumen como la primera compilación de narrativa breve italiana publicada en Cuba de la que no existe, en cambio, ninguna reedición posterior (Bello, 2019, p. 145). La casi totalidad de los cuentos reunidos allí fue traducida por Bertarelli; sin embargo, aparece también, citada en algunas presentaciones previas a los relatos, la prestigiosa traductora española María Esther Benítez. Los textos por ella traducidos son "Los siete mensajeros", de Buzzati; "Una velada inolvidable", de Brancati; "Giufá", de Sciascia, y "Un par de gafas", de Ortese. Aunque no quedara declarada la fuente, estas traducciones probablemente fueron extraídas del volumen Relatos italianos del siglo $\mathrm{XX}$, publicado por Alianza Editorial en 1974 (460 pp.), una antología a cargo de Guido Davico Bonino (prólogo, selección y notas) y traducida por María Esther Benítez y José Antonio Sánchez Ferlosio.

Hasta el momento Bertarelli se había encargado exclusivamente de trabajar con obras teóricas y literarias del siglo $\mathrm{xx}$, sin embargo, en 1981 se dio a conocer en Cuba la novela decimonónica Confesiones de un italiano, de Ippolito Nievo, que podríamos definir como su capolavoro de traducción, una obra que se publicó gracias a su propuesta personal. La edición que sirve de referencia para este volumen, que a su vez representa la primera traducción en lengua española, es la de Feltrinelli (Milán, 1960). El título compuesto por dos tomos lleva un exquisito prólogo de Graziella Pogolotti en el que aborda elementos del autor y su época:

La elaboración de las Confesiones de un italiano responde a un proyecto ambicioso que puede ser resumido provisionalmente como el de dilucidar, mediante el empleo de la ficción, las razones que hacen necesaria la consolidación de la unidad y de la independencia italianas. (Pogolotti, 1981, p. Ix) 
El estudio preliminar de Pogolotti, que bien merece una reedición independiente junto a otros de sus prólogos, como sugiriera Luisa Campuzano (2006), abarca detalles del proyecto de liberación nacional en el que estaba implicado el autor, cuyo pensamiento político se inspiraba en Giuseppe Mazzini. Se explican además con suma sagacidad aspectos propiamente narratológicos: "En otra circunstancia y en un clima romántico, Ippolito Nievo se plantea sobrepasar las limitaciones de la novela histórica y de la novela de la formación del hombre. La naturaleza del proyecto determina la estructura interna de la obra" (Pogolotti, 1981, p. xx). Confesiones de un italiano fue la última obra italiana traducida por Giannina Bertarelli; esa edición aunaba el trabajo de dos grandes amigas; será por eso, además de la preferencia por este clásico de la literatura, que la traductora le tenía tan alta estima a esta publicación.

Un caso aparte de traducción es el que implica a la escritora italiana de origen cubano Alba de Céspedes, que se había propuesto escribir desde finales de los años sesenta un libro de memorias relacionado con la historia de la isla, la cual se mezclaba con la de su propia familia. A raíz de este proyecto literario que recibió el apoyo inmediato de dirigentes e intelectuales de la Revolución, la novelista emprendió diversos viajes durante los años setenta con el fin de escribir una obra que, sin embargo, permanecería inconclusa. Alba de Céspedes contactó a Giannina Bertarelli — quizás a través de Rolando Rodríguez (entonces director del Instituto Cubano del Libro hasta 1976, cuando sería designado viceministro de Cultura) para una traducción simultánea al español del libro que estaba escribiendo y que titularía Con grande amore. ${ }^{16}$

16 Se trata de una traducción simultánea, pues la autora enviaba a traducir fragmentos de su novela en la medida en que escribía.
Durante largos años se mantuvo la correspondencia entre ambas intelectuales inmersas en este proyecto que con el tiempo fue decreciendo hasta frustrarse en 1997 con el deceso de la novelista en París. Los fragmentos del libro de Alba de Céspedes traducidos por Bertarelli asumieron la denominación de archivo Traduzione Giannina, como la propia escritora definiera en la cubierta de la carpeta donde los conservaba. En el 2011 se publicaron por primera vez algunos de estos fragmentos, gran parte de estos pertenecientes a entrevistas que Alba de Céspedes dirigiera a diversos cubanos con el fin de reunir voces plurales en su libro autobiográfico. La selección de estos textos quedó a cargo de Luisa Campuzano y Anele Arnautó Trillo, editora del volumen, y se muestra en el apéndice de Con gran amor (La Habana: Ediciones Unión, 2011). Esa labor iniciada por Giannina Bertarelli durante los años setenta fue continuada por la traductora cubana Mayerín Bello, quien se ocupó de trasladar la novela inacabada de Alba de Céspedes, completamente inédita hasta la edición preparada por Monica Cristina Storini para el volumen Romanzi (Milán: Mondadori, 2011). Según apunta la propia Storini:

La "Traducción Giannina" consta de 137 cuartillas; las tres primeras contienen un listado manuscrito y dos copias mecanografiadas (la segunda en papel carbón) de los Fragmentos traducidos (Pezzi tradotti), con la indicación de su extensión según la cantidad de páginas que ocupan [...]. (Storini, 2011, p. 48)

Estructurado, salvo rarísimas excepciones, a partir de personajes que cuentan sus propias experiencias de vida y de desempeño político, el texto se presenta como una narración en primera persona que ha resuelto, con la alternancia de parlamentos y con la selección de la forma de la escena, la difícil relación entre el presente autobiográfico y el pasado histórico. El vínculo entre la memoria y el presente histórico se funde, sin embargo, en el reconocimiento de una continuidad entre las guerras de independencia y la Revolución cubana [...]. (Storini, 2011, pp. 50-51) 
Se trata de un material valioso que habla de la génesis de un libro que habría sido la gran obra cubana de la novelista Alba de Céspedes. La colaboración de Giannina Bertarelli en este proyecto de traducción simultánea propiciaba que el texto contara con pertinentes lectores cubanos que pudieran servir de asesores literarios. De otra manera no se explica el porqué de la inmediata traducción de una obra que distaba mucho de su estación final. Este caso constituye, a todas luces, un singular diálogo transatlántico que pertenece a una serie de episodios vinculados con la historia de la escritura de Con grande amore.

\section{A modo de conclusión}

En 1984, Giannina Bertarelli publicó su última traducción literaria, esta vez del francés, Kamouraska (296 pp.), de la escritora canadiense Anne Hébert, para la Editorial Arte y Literatura. Por esos años Cuba aprobó la ley de derecho de autor; Bertarelli se dedicaría entonces a las colaboraciones periodísticas para Italia desde esa isla que había elegido por estancia definitiva.

$\mathrm{Su}$ trayectoria como legítima traductora, forjada de modo autodidacta, había quedado consagrada desde aquellas colaboraciones con Pensamiento Crítico en los años sesenta y su pertenencia luego al Instituto Cubano del Libro hasta la década de los ochenta. La complejidad y diversidad de las temáticas traducidas, la destreza en el dominio de los idiomas, aprendidos de modo empírico, así como el deber traducir hacia una lengua extranjera títulos que no se conocían en español son las claves para apreciar el oficio de una mujer de la cual habría de decirse mucho más en futuro.

La reconstrucción primera de este corpus define las líneas estilísticas abordadas en los trabajos de traducción de Giannina Bertarelli: textos en prosa de matriz teórico-crítica o literaria. Presentar aquí como parte de un bosquejo inicial este conjunto de títulos, en particular, los pertenecientes a obras literarias italianas anuncia desde ya la necesidad de continuar y profundizar en aspectos lingüísticos que caracterizan de modo intrínseco el trabajo acometido. El estudio a fondo, por ejemplo, de Confesiones de un italiano, de Ippolito Nievo, en comparación con otras traducciones al español o de un examen de la edición per se, es una de las principales demandas que surgen a partir de esta investigación. Si bien es cierto que la mayoría de los libros traducidos responden a un encargo, a una decisión institucional, puede apreciarse en algunos casos la participación de Bertarelli en la propuesta de obras que eran de su gusto y estima. Así, hay que destacar la incorporación al catálogo de la Editorial Arte y Literatura del propio Nievo, de Fenoglio, y de otros autores cardinales del siglo xx que forman parte de la antología Una noche del 43 y otros relatos. Son estos resultados esenciales de la contribución traductológica de Giannina Bertarelli y de su noble afán por difundir la literatura de su tierra natal en la isla que la acogió.

La biografía intelectual de Giannina Bertarelli reseñada aquí presenta el caso de una viajera intrépida, de una feminista avant la lettre, de una emigrante italiana que se volvió cubana y, además, genuina mediadora cultural. Sin embargo, tanto su vida como su obra han quedado circunscritas a una minoría, la de mujeres emigrantes traductoras, y como minoría ha sido relegada con frecuencia al silencio. Investigar sobre el itinerario biográfico y bibliográfico de esta figura hoy define un espacio propio que le pertenece en el marco de la historia de la cultura cubana contemporánea en valioso diálogo con Italia. El rescate de la historia de las mujeres escritoras y —añadiríatraductoras, sigue siendo una tarea de rigor para las ciencias sociales, en particular, para 
los estudios literarios que desde hace algunas décadas miran con atención el rol femenino en la cultura del siglo xx. Como metodología establecida por varias estudiosas italianas, la biografía intelectual ha hecho posible un "redescubrimiento social" de personalidades literarias silenciadas, como Fausta Cialente, Alba de Céspedes, Paola Masino y Gianna Manzini, que a su vez ha generado una difusión mayor de sus obras y un despertar de la crítica. Es ese el mejor augurio de estas páginas dedicadas a una italiana de nombre Giannina Bertarelli, que traducía en La Habana.

\section{Referencias}

ABC. (2009). Editan por primera vez en español las memorias del indio Gerónimo. $A B C$. https://www.abc.es/ cultura/libros/abci-editan-primera-espanol-memorias-indio-geronimo-200908220300-1023529299782_noticia.html

Althusser, L. (1969). Lenin y la filosofía. (G. Bertarelli, Trad.). Pensamiento Crítico, (2930), 120-153.

Apresian, I. D. (1985). Ideas y métodos de la lingüistica estructural contemporánea (G. Bertarelli, Trad.). Ciencias Sociales.

Bálazs, B. (1980). La estética del film (G. Bertarelli, Trad.). Editorial Arte y Literatura.

Bassani, G., Compagnone, L.; Rea, D. ; Festa Campanile, P. ... Berto, G. (1981). Una noche del 43 y otros relatos (G. Bertarelli, Trad.). Editorial Arte y Literatura.

Bello, M. (2019). Golondrinas que hacen discretos veranos: antologías contemporáneas de cuentos italianos y cubanos. En M. C. Secci (ed.), Miscelánea. Studi traduttologici, linguistici e letterari su America Latina e Caraibi (pp. 143-164). Unicapress. https://doi.org/10.13125/unicapress.978-88-3312-008-9
Bertarelli, G. (1981). Al lector (Prólogo). En Bassani et al., Una noche del 43 y otros relatos (G. Bertarelli, Trad.). Editorial Arte y Literatura.

Biblioteca Nacional de Cuba "José Martí" (1982). Bibliografia cubana. Biblioteca Nacional de Cuba "José Martí".

Biblioteca Nacional de Cuba "José Martí" (1985). Bibliografía cubana. Biblioteca Nacional de Cuba "José Martí".

Bravo, G. M. (1976). Los socialistas anteriores a Marx (G. Bertarelli, P. Arce y M. Díaz Godoy, Trads.). Ciencias Sociales.

Campuzano, L. (2006). Una mente privilegiada. La Jiribilla [Transcripción discurso en Instituto Cubano del Libro, documento html]. https://www.lajiribilla. co.cu/2006/n291_12/291_11.html

Capolongo, D. (ed.) (2010). Emigrazione e presenza italiana in Cuba. Diaconia Grafica \& Stampa.

Carpentier, A. (1984). Ensayos. Letras Cubanas.

Davico Bonino, G. (ed.) (1974). Relatos italianos del siglo XX (M. E. Benítez y J. A. Sánchez Ferlosio, Trads.). Alianza Editorial.

De Céspedes, A. (2011). Con gran amor (M. Bello, Trad.). Ediciones Unión.

Di Nicola, L. (2012). Mercurio. Storia di una rivista 1944-1948. Mondadori.

Dutshke, R. (1968). Las contradicciones del capitalismo tardío, los estudiantes antiautoritarios y su relación con el Tercer Mundo (G. Bertarelli, Trad.). Pensamiento Crítico, (20), 67-147.

Fenoglio, B. (1980). Los veintitrés días de la ciudad de Alba (G. Bertarelli, Trad.). Editorial Arte y Literatura.

Garzia, A. y Fleites, A. (1997). Cuba, cultura. Viaggio nell'identità di un'isola. Teti Editore.

Gerónimo (1982). El indio Gerónimo: memorias: recopiladas por $S$. M. Barrett (G. Bertarelli, Trad.). Ciencias Sociales. 
Giap, V. N. (1975). Armar a las masas revolucionarias, construir el ejército popular (G. Bertarelli, Trad.). Ciencias Sociales.

Ginzburg, N. (2016). Las tareas de casa y otros ensayos. Lumen.

Gorresio, V. (1953). Avventure di giornalisti in un paese che non li ama. La Stampa, IX(217), 5 .

Grant, M. (2003). Oficio de remembranza con Graziella Pogolotti. Opus Habana, VII(3), 18-29.

Hébert, A. (1984). Kamouraska (G. Bertarelli, Trad.). Editorial Arte y Literatura.

Hewitt, N. (2007). Images of Cuba in France in the 1960s: Sartre's 'Ouragan sur le sucre'. Sartre Studies International, 13(1), 62-73. https://www.jstor.org/stable/23511188

Kohan, N. (1993). Cuba y el pensamiento crítico: entrevista con Fernando Martínez Heredia (Entrevista en blog). La Haine. https://www.lahaine.org/mundo.php/cuba-y-el-pensamiento-critico

Landolfi, T. (1980). Las dos solteronas (G. Bertarelli, Trad.). Editorial Arte y Literatura.

Martínez Heredia, F. (1970). Althusser y el marxismo. Pensamiento Crítico, (36), 210215.

Martínez Heredia, F. (2008). A cuarenta años de Pensamiento Crítico. Crítica y Emancipación. Revista Latinoamericana de Ciencias Sociales, (1), 237-250.

Marx, K. (1975). Cartas a Kugelmann (G. Bertarelli, Trad.). Ciencias Sociales.

Micheli, M. (1972). Las vanguardias artísticas del siglo XX (G. Bertarelli, Trad.). Instituto Cubano del Libro.

Nievo, I. (1981). Confesiones de un italiano (G. Bertarelli, Trad.). Editorial Arte y Literatura.

Ortiz, F. (1905). Las simpatías de Italia por los mambises cubanos. Marsella.

Ortiz, F. (1909). Los mambises italianos (apuntes para la historia cubana). Imprenta Cuba y América.
Ortiz, F. (1944). Italia y Cuba. Imprenta Atalaya.

Ouellete, F. (1989). Edgard Varèse (G. Bertarel1i, Trad.). Arte y Literatura.

Paolucci, G. (2007). Sartre's humanism and the Cuban revolution. Theory and Society, 36(3), 245-263. https://doi.org/10.1007/ s11186-007-9031-3

Pertierra Serra, E. (2000). Italianos por la libertad de Cuba. Editorial José Martí.

Pogolotti, G. (1981). Al lector. En I. Nievo, Confesiones de un italiano (pp. VII-XXIII). Arte y Literatura.

Pogolotti, G. (2012). Órbita de Virgilio. La Jiribilla. https://www.rebelion.org/noticia. php?id $=142900$

Pogolotti, G. (2018). Más sobre el ICL y sus traductores. Giannina, persona, contextos. Revolución y Cultura, (1), 24-25.

Ponce Suárez, V. (2007). Una mirada métrica a la revista Pensamiento Crítico. Bibliotecas. Anales de investigación, (2), 102-138.

Ponce Suárez, V. (2019). La présence française dans la revue Pensamiento Crítico. Lettres de Cuba, (12). http://www.lettresdecuba. cult.cu/?q=articles/la-pr\%C3\%A9sencefran $\% \mathrm{C} 3 \% \mathrm{~A} 7$ aise-dans-la-revue-pensamiento-cr\%C3\%ADtico-fin.html

Rodari, G. (1980). Aventuras de Cebollino (G. Bertarelli, Trad.). Gente Nueva.

Rosselló, R. (2019). Presencia italiana en Cuba (1492-1902). Xlibris Us.

Rubini, F. (2019). Fausta Cialente. La memoria e il romanzo. Mondadori.

Sánchez Guevara, O. (2018). Un enclave intercultural en La Habana: Mis experiencias en el Departamento de Traducciones del ICL. Revolución y Cultura, (1), 21-23.

Sartre, J. P. (1960a). Huracán sobre el azúcar. Ministerio de Relaciones Exteriores de Cuba.

Sartre, J. P. (1960b). Ideología y revolución (J. J. A., Trad.). Lunes de Revolución, (51), 3-9. 
Sartre, J. P. (1967). El pensamiento político de Patricio Lumumba. Pensamiento Crítico, (2-3), 50-92.

Sartre, J. P. (1968). El intelectual frente a la Revolución (G. Bertarelli, Trad.). Pensamiento Crítico, (20), 191-205.

Sartre, J. P. (1969). África del Sur: centro del fascismo. Pensamiento Crítico, (32), 129-134.

Sciascia, L. (1977). El día de la lechuza (G. Bertarelli, Trad.). Editorial Arte $\mathrm{y}$ Literatura.
Storini, M. C. (2011). Con gran amor y la "Traducción Giannina". En A. de Céspedes, Con gran amor (pp. 41-52). Ediciones Unión.

Venuti, L. (1995). The translator's invisibility: A history of translation. Routledge.

Zancan, M. (ed.) (2005). Scrittrici e intelletuali del Novecento. Alba de Céspedes. Approfondimenti. Mondadori.

Cómo citar este artículo: González, I. (2020). Giannina Bertarelli, una italiana que traducía en La Habana. Reflexiones sobre vida y obra. Mutatis Mutandis. Revista Latinoamericana de Traducción, 13(2), 300-318 https://doi.org/10.17533/udea.mut.v13n2a05 